\title{
Recent KLOE results on Kaon Physics
}

\author{
Aleksander Gajos* on behalf of the KLOE-2 Collaboration \\ Jagiellonian University, Cracow \\ E-mail: aleksander.gajos@uj.edu.pl
}

The KLOE experiment at the DA $\Phi N E \phi$-factory of the INFN Frascati Laboratory collected data corresponding to $2.5 \mathrm{fb}^{-1}$ of integrated luminosity. Neutral kaon pairs produced in phi-meson decays offer unique possibilities to perform tests of fundamental discrete symmetries. The entanglement of the two kaons is exploited to search for possible violation of CPT symmetry and Lorentz invariance in the context of the Standard-Model Extension (SME) framework. A new approach to the analysis of $\phi \rightarrow K_{S} K_{L} \rightarrow \pi^{+} \pi^{-}, \pi^{+} \pi^{-}$events has been adopted allowing us to independently measure all four CPT violating parameters $\Delta a_{\mu}$ appearing for neutral kaons in the SME. The final KLOE results on $\Delta a_{\mu}$ will be presented. These are presently the most precise measurements in the quark sector of the SME. We also shortly discuss the perspectives for a new measurement using the KLOE-2 apparatus equipped with a new inner tracker. The measurement of the absolute BR of the $K^{+} \rightarrow 3 \pi(\gamma)$ decay completes the KLOE program of precise and fully inclusive kaon dominant BR measurements. The most recent result, BR $\left(K^{+} \rightarrow \pi^{+} \pi^{+} \pi^{-}\right)=(5.56 \pm 0.20) \%$ (Chiang et al.), dates back to more than 30 years ago. We use a normalization sample of events tagged by $K \rightarrow \mu \nu$ and $K \rightarrow \pi \pi^{0}$ decays and require at least two reconstructed tracks (pion candidates). Finally the number of $K \rightarrow 3 \pi(\gamma)$ decays is counted from the missing mass spectrum of the third pion to extract the branching ratio. The final KLOE result on the $\operatorname{BR}\left(K^{+} \rightarrow \pi^{+} \pi^{+} \pi^{-}(\gamma)\right)$ is also presented.

XIIth International Conference on Heavy Quarks \& Leptons 2014

25-29 August 2014

Schloss Waldthausen, Mainz, Germany

\footnotetext{
* Speaker.
} 


\section{The KLOE experiment}

KLOE (K Long Experiment) is located at one of the two interaction regions of the DA $\Phi N E$ collider operating at the National Laboratory of Frascati, Italy. At DA $\Phi$ NE electrons and positrons collide with $\sqrt{s} \approx 1020 \mathrm{MeV}=m_{\phi}$, producing predominantly $\phi$ mesons being almost at rest $\left(p_{\phi} \approx\right.$ $12 \mathrm{MeV} / \mathrm{c})$. The $\phi$ mesons decay into pairs of kaons, either neutral or charged, with branching ratios of $34 \%$ and $49 \%$, respectively, allowing the KLOE detector which surrounds the decay point to study a broad range of kaons physics. Production of the kaons in pairs provides two unique features of the KLOE experiment, i.e. the possibility of tagging kaon states and quantum entanglement. The former means that detection of charged or neutral kaon guarantees the presence of its corresponding orthogonal state in the same event, which is used to obtain pure beams of charged or neutral kaons e.g. for branching ratio measurements. The second feature is the quantum coherence of the two kaon states which allows for measurements using quantum interferometry.

KLOE is a barrel-shaped detector composed mainly of a cylindrical drift chamber with a radius of $2 \mathrm{~m}$ and a lead-scintillating fiber sampling electromagnetic calorimeter which surrounds the drift chamber covering $98 \%$ of full solid angle. While the size of detector was chosen so as to capture about $40 \%$ of the long-lived kaon decays, the $K_{s}$ decays are contained within a spherically-shaped beam pipe with $10 \mathrm{~cm}$ radius designed to avoid $K_{S}$ regeneration on the BP material. The detector collected a total of about $2.5 \mathrm{fb}^{-1}$ of data in two runs in the years 2001-2002 and 2004-2005, with loose trigger conditions which allow for a broad physics program.

\section{CPT and Lorentz symmetry test with entangled neutral kaons}

According to the Standard Model Extension (SME) [1] and the Anti-CPT theorem [2], CPT violation should appear together with breaking of Lorentz invariance. The SME parameters related to the kaon sector can be estimated using the methods of quantum interferometry. The amplitude of two entangled neutral kaons decaying into final states $f_{1}$ and $f_{2}$ in times differing by $\Delta \tau$ can be expressed as below:

$$
I_{f_{1} f_{2}}(\Delta \tau)=C e^{-\Gamma|\Delta \tau|}\left[\left|\eta_{1}\right|^{2} e^{\frac{\Delta \Gamma}{2} \Delta \tau}+\left|\eta_{2}\right|^{2} e^{-\frac{\Delta \Gamma}{2} \Delta \tau}-2 \Re e\left(\eta_{1} \eta_{2}^{*} e^{-i \Delta m \Delta \tau}\right)\right],
$$

where $\Delta \Gamma=\Gamma_{S}-\Gamma_{L}, \Gamma=\left(\Gamma_{S}+\Gamma_{L}\right) / 2, C$ is a normalization constant, $\eta_{j}=\frac{\left\langle f_{j}|T| K_{L}\right\rangle}{\left\langle f_{j}|T| K_{S}\right\rangle}$ and the last term in eq. 2.1 arises from quantum interference of kaon states. If both final states are chosen as $\pi^{+} \pi^{-}, \eta_{1}$ and $\eta_{2}$ can be expressed in terms of the parameters $\varepsilon_{K}$ and $\delta$. In the framework of SME, however, $\delta$ is not constant and exhibits a modulation dependent on the direction of the kaon according to:

$$
\delta \simeq i \sin \phi_{S W} e^{i \phi_{S W}} \gamma_{K}\left(\Delta a_{0}-\vec{\beta}_{K} \Delta \vec{a}\right) / \Delta m
$$

where $\phi_{S W}$ is the superweak phase, $\gamma_{K}$ and $\vec{\beta}_{K}$ are the kinematic parameters of the corresponding kaon and $\Delta a_{0}, \Delta \vec{a}$ are the coefficients violating CPT and Lorentz symmetries in the SME. The $\Delta a_{\mu}$ parameters may be extracted from a measurement of the $\delta$ parameter direction and energy dependence.

Since for the $\phi \rightarrow K_{S} K_{L} \rightarrow \pi^{+} \pi^{-} \pi^{+} \pi^{-}$process the two kaons travel with almost opposite momenta in the laboratory frame, their associated $\delta$ parameters should differ and the two relations:

$$
\eta_{1}=\varepsilon_{K}-\delta\left(\vec{p}_{K^{1}}\right), \quad \eta_{2}=\varepsilon_{K}-\delta\left(\vec{p}_{K^{2}}\right)
$$


lead to an effect on the distribution given in eq. 2.1. In order to observe it, the $\delta$ direction dependence is expressed in the reference frame of fixed stars, which also introduces its dependence on sidereal time.

The strategy of selection of $\phi \rightarrow K_{S} K_{L} \rightarrow \pi^{+} \pi^{-} \pi^{+} \pi^{-}$events relies on a requirement of two reconstructed vertices with two associated tracks each and a set of cuts limiting the missing kinematic variables as the charged pion tracks provide full kinematic information on the signal events. Moreover, the considered time difference between kaon decays is limited to $\Delta \tau \in\left[-12 \tau_{S} ; 12 \tau_{S}\right]$ in order to avoid $K_{L}$ regeneration on the beam pipe material. The kaon decay length reconstruction is improved by means of a global kinematic fit. The residual contamination of the selected sample consists of kaon regeneration (2\%) and direct $\phi \rightarrow \pi^{+} \pi^{-} \pi^{+} \pi^{-}$decays (0.5\%).

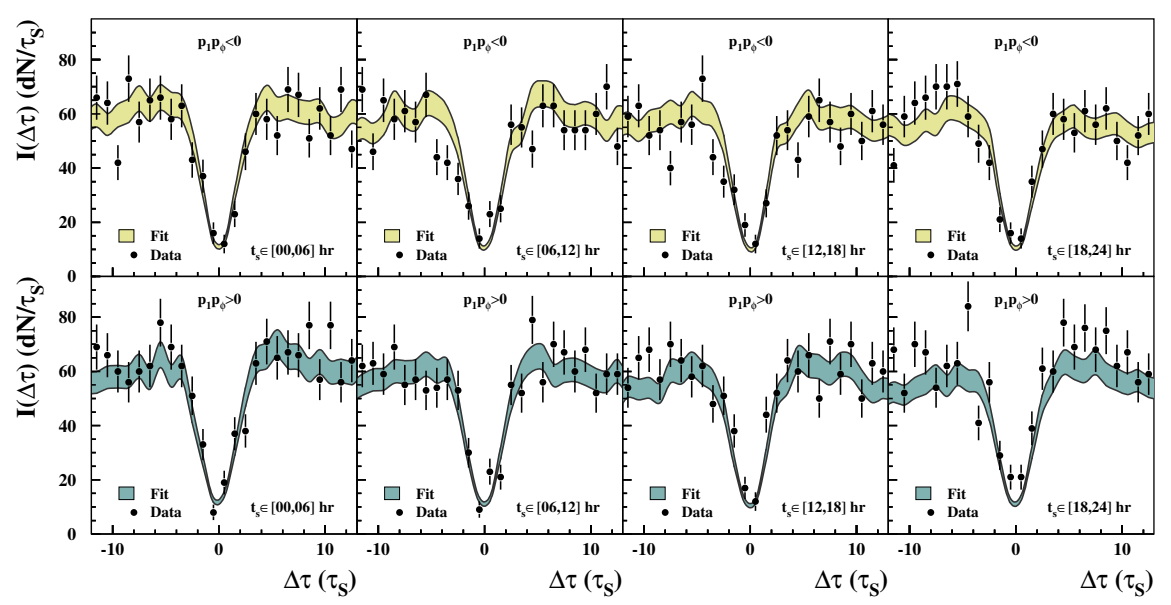

Figure 1: Results of the fit (color bands) to the double decay intensity distributions for data (black points) divided into 4 bins of sidereal time and two angular bins [3].

In order to observe the effect of direction-dependent modulation of $\delta$ on the intensity from eq. 2.1, the two kaons are ordered by the $z$-component of their momentum and the selected dataset is divided into two angular samples - one where a kaon with $\cos \left(\theta_{\vec{p}}\right)>0$ in the detector frame has $\overrightarrow{p_{K}} \cdot \overrightarrow{p_{\phi}}>0$ and one with $\overrightarrow{p_{K}} \cdot \overrightarrow{p_{\phi}}<0$. The data is further divided into 4 samples of sidereal time ranges which results with 8 samples of data for which the decay amplitude distributions are simultaneously fit to extract the $\Delta a_{\mu}$ parameters. The result of the fit is shown in fig. 1 . The obtained values of the SME parameters are the following:

$$
\begin{aligned}
& \Delta a_{0}=\left(-6.0 \pm 7.7_{\text {stat }} \pm 3.1_{\text {syst }}\right) \times 10^{-18} \mathrm{GeV}, \\
& \Delta a_{X}=\left(0.9 \pm 1.5_{\text {stat }} \pm 0.6_{\text {syst }}\right) \times 10^{-18} \mathrm{GeV}, \\
& \Delta a_{Y}=\left(-2.0 \pm 1.5_{\text {stat }} \pm 0.5_{\text {syst }}\right) \times 10^{-18} \mathrm{GeV}, \\
& \Delta a_{Z}=\left(3.1 \pm 1.7_{\text {stat }} \pm 0.5_{\text {syst }}\right) \times 10^{-18} \mathrm{GeV} .
\end{aligned}
$$

Reaching the expected sensitivity at the level $\mathrm{O}\left(10^{-18} \mathrm{GeV}\right)$, these results are presently the most precise measurements in the quark sector of the SME [3]. As the statistical uncertainties are dominating, there are prospects for improvement of this result with higher statistics. 


\section{Measurement of the absolute branching ratio of $K^{+} \rightarrow \pi^{+} \pi^{-} \pi^{+}(\gamma)$}

The measurement of $\operatorname{BR}\left(K^{+} \rightarrow \pi^{+} \pi^{-} \pi^{+}(\gamma)\right)$ completed the KLOE program of precise and fully inclusive measurements of $K^{ \pm}$dominant branching ratios. It is the last result necessary for a global fit of $K^{ \pm}$branching ratios with KLOE results but it is also an input required for the CUSP analysis done by the NA48 experiment to extract the $\pi \pi$ phase [4]. Moreover, the previous experimental result dates back to 1972 and provides no information on radiation cut-offs [5].

The analysis is performed using a sample of $174 \mathrm{fb}^{-1}$ of KLOE data. Two normalization samples are selected to contain self-triggering decays $K^{-} \rightarrow \mu^{-} \nu$ and $K^{-} \rightarrow \pi^{-} \pi^{0}$ which indicate the presence of $\mathrm{K}^{+}$in the second hemisphere of the detector thanks to the tagging possibility at KLOE. The $\mathrm{K}^{+}$decays are chosen for the BR measurement with tagging by $\mathrm{K}^{-}$in order to minimize background from nuclear interactions whose cross-section at $p \approx 100 \mathrm{MeV}$ is a factor $10^{3}$ smaller for $K^{+}$. The track of tagging kaon is reconstructed in the DC and extrapolated backwards to obtain a virtual path of the signal kaon. In order to optimize the quality of the track reconstruction, kaons decaying before the Drift Chamber inner wall are selected, so that only their decay products enter the DC which minimizes the number of present tracks. Presence of at least two reconstructed tracks of pion candidates in an event is required. Number of signal events is obtained through a fit to the invariant mass spectrum of the third missing pion, separately for each of the normalization samples.
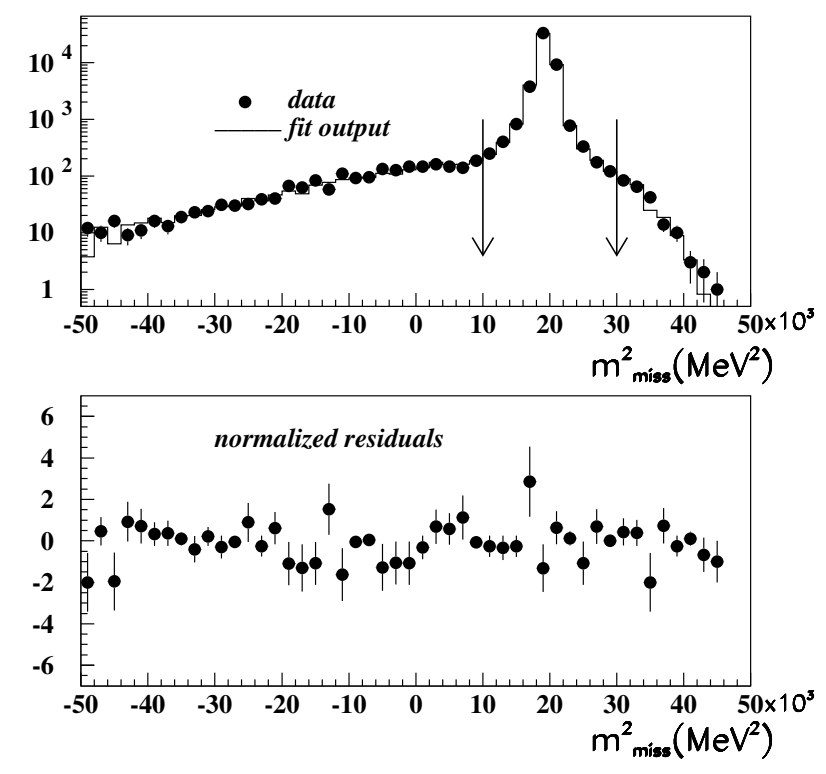

Figure 2: Fit of the MC to data for the missing mass spectrum obtained for the normalization sample tagged by $K^{-} \rightarrow \mu^{-} v$ decays.

Fig. 2 shows a result of the fit for the sample tagged by $K^{-} \rightarrow \mu^{-} v$. Signal events $(K \rightarrow 3 \pi(\gamma))$ are counted in the missing mass region $10000 \mathrm{MeV}^{2}<m_{\text {miss }}^{2}<30000 \mathrm{MeV}^{2}$. Finally, the branching 
ratio is determined as the efficiency-corrected ratio:

$$
\mathrm{BR}\left(K^{+} \rightarrow \pi^{+} \pi^{-} \pi^{+}\right)=\frac{N_{K \rightarrow 3 \pi}}{N_{t a g}} \times \frac{1}{\varepsilon_{s e l} C_{T B} C_{f} C_{c r v}},
$$

where $N_{\text {tag }}$ is the total size of the normalization sample, $\varepsilon_{s e l}$ is the selection efficiency and $C_{T B}, C_{f}$ and $C_{c r v}$ are corrections for tag bias, machine background filter and cosmic ray veto, respectively.

The following results were obtained independently for the two normalization samples:

$$
\begin{aligned}
& \left.B R\left(K^{+} \rightarrow \pi^{+} \pi^{-} \pi^{+}(\gamma)\right)\right|_{T a g K_{\mu 2}}=0.05552 \pm 0.00034_{\text {stat }} \pm 0.00034_{\text {syst }}, \\
& \left.B R\left(K^{+} \rightarrow \pi^{+} \pi^{-} \pi^{+}(\gamma)\right)\right|_{T a g K_{\pi 2}}=0.05587 \pm 0.00053_{\text {stat }} \pm 0.00033_{\text {syst }},
\end{aligned}
$$

and were finally averaged yielding the final result [6]:

$$
B R\left(K^{+} \rightarrow \pi^{+} \pi^{-} \pi^{+}(\gamma)\right)=0.05565 \pm 0.00031_{\text {stat }} \pm 0.00025_{\text {syst }},
$$

whose precision is better than the previous measurement [5] by a factor of 5 .

Moreover, the above result was used along with other $K^{ \pm}$branching ratios and life-time determined by KLOE to perform a global fit with a unitary constraint. Results of the fit are presented in table 3.

\begin{tabular}{llccccccc}
\hline \hline Parameter & Value & \multicolumn{6}{c}{ Correlation coefficients } \\
\hline $\operatorname{BR}\left(K_{\mu 2}^{ \pm}\right)$ & $0.6372(11)$ & & & & & & \\
$\operatorname{BR}\left(K_{\pi_{2}}^{ \pm}\right)$ & $0.2070(9)$ & 0.55 & & & & & \\
$\operatorname{BR}\left(\pi^{ \pm} \pi^{-} \pi^{+}\right)$ & $0.0558(4)$ & -0.23 & -0.05 & & & & \\
$\operatorname{BR}\left(K_{e 3}^{ \pm}\right)$ & $0.0498(5)$ & 0.42 & -0.15 & 0.06 & & & \\
$\operatorname{BR}\left(K_{\mu 3}^{ \pm}\right)$ & $0.0324(4)$ & -0.39 & 0.14 & -0.05 & -0.58 & & \\
$\operatorname{BR}\left(\pi^{ \pm} \pi^{0} \pi^{0}\right)$ & $0.01764(25)$ & -0.13 & 0.05 & -0.02 & 0.04 & -0.04 & \\
$\tau_{K^{ \pm}}(\mathrm{ns})$ & $12.344(29)$ & 0.20 & 0.19 & -0.14 & 0.05 & -0.04 & 0.02 \\
\hline \hline
\end{tabular}

Table 1: Results of the global fit of $K^{ \pm}$branching ratios and life-time with KLOE measurements [6].

\section{Conclusions and prospects for KLOE-2}

The KLOE experiment provided a variety of Physics results, including interferometric tests of fundamental symmetries such as the CPT and Lorentz symmetry but also extensive measurements of kaon properties, finalized by the measurement of $\operatorname{BR}\left(K^{+} \rightarrow \pi^{+} \pi^{+} \pi^{-}(\gamma)\right)$. The results on the $\Delta a_{\mu}$ parameters are the most precise results in the SME quark sector to date and several orders of magnitude better than for other meson systems. The $\operatorname{BR}\left(K^{+} \rightarrow \pi^{+} \pi^{+} \pi^{-}(\gamma)\right)$ measurement along with previous KLOE results constitutes a consistent description of the $K^{ \pm}$properties as was shown by the global unitary fit.

Recently an upgrade of KLOE has been performed. The improved KLOE-2 detector was equipped with several new subdetectors. Two pairs of new calorimeters were mounted at small angles around the beam pipe to increase acceptance for photons coming from the interaction point 
[7, 8]. Moreover, the region around the interaction point was instrumented with a novel cylindrical GEM inner tracker which increases acceptance for low- $p_{T}$ tracks and improves vertexing [9]. The improved vertex resolution for $K \rightarrow \pi^{+} \pi^{-}$is expected to increase the time resolution in the interferometric CPT and Lorentz symmetry test, and correspondingly the sensitivity on the $\Delta a_{\mu}$ parameters by a factor of 2, as well as on other QM and CPT violation parameters [10].

Along with the KLOE-2, the DAФNE collider was upgraded to utilize a new crabbed-waist collision scheme enabling it to provide higher luminosity. KLOE-2 is now starting operations with a plan to collect data corresponding to an integrated luminosity $\mathrm{O}\left(10 \mathrm{fb}^{-1}\right)$ in 2-3 years.

\section{Acknowledgments}

This work was supported in part by the EU Integrated Infrastructure Initiative Hadron Physics Project under contract number RII3-CT- 2004-506078; by the European Commission under the 7th Framework Programme through the 'Research Infrastructures' action of the 'Capacities' Programme, Call: FP7-INFRASTRUCTURES-2008-1, Grant Agreement No. 227431; by the Polish National Science Centre through the Grants No. DEC-2011/03/N/ST2/02641, 2011/01/D/ST2/00748, 2011/03/N/ST2/02652, 2013/08/M/ST2/00323, 2013/11/B/ST2/04245, and by the Foundation for Polish Science through the MPD programme and the project HOMING PLUS BIS/2011-4/3.

\section{References}

[1] V. A. Kostelecky, Formalism for CPT, T, and Lorentz violation in neutral meson oscillations, Phys. Rev. D 64 (2001) 076001 [hep-ph/0104120].

[2] O. W. Greenberg, CPT violation implies violation of Lorentz invariance, Phys. Rev. Lett. 89 (2002) 231602 [hep-ph/0201258].

[3] D. Babusci et al. [KLOE-2 Collaboration], Test of CPT and Lorentz symmetry in entangled neutral kaons with the KLOE experiment, Phys. Lett. B 730 (2014) 89 [arXiv:1312.6818 [hep-ex]].

[4] J. R. Batley et al. [NA48/2 Collaboration], Observation of a cusp-like structure in the $\pi^{0} \pi^{0}$ invariant mass distribution from $K^{ \pm} \rightarrow \pi^{ \pm} \pi^{0} \pi^{0}$ decay and determination of the $\pi \pi$ scattering lengths, Phys. Lett. B 633 (2006) 173 [hep-ex/0511056].

[5] I. H. Chiang, J. L. Rosen, S. Shapiro, R. Handler, S. Olsen and L. Pondrom, $K^{+}$Decay in Flight, Phys. Rev. D 6 (1972) 1254.

[6] D. Babusci et al. [KLOE/KLOE-2 Collaboration], Measurement of the absolute branching ratio of the $K^{+} \rightarrow \pi^{+} \pi^{-} \pi^{+}(\gamma)$ decay with the KLOE detector, Phys. Lett. B 738 (2014) 128 [arXiv:1407.2028 [hep-ex]].

[7] M. Cordelli, G. Corradi, F. Happacher, M. Martini, S. Miscetti, C. Paglia, A. Saputi and I. Sarra et al., QCALT: A Tile calorimeter for KLOE-2 experiment, Nucl. Instrum. Meth. A 617 (2010) 105 [arXiv:0906.1133 [physics.ins-det]].

[8] M. Cordelli, E. Dane, S. Giovannella, M. Gatta, F. Happacher, A. Luca, S. Miscetti and A. Saputi et al., CCALT: A Crystal calorimeter with timing for the KLOE-2 upgrade, Nucl. Instrum. Meth. A 718 (2013) 81. 
[9] A. Balla, G. Bencivenni, S. Cerioni, P. Ciambrone, E. De Lucia, G. De Robertis, D. Domenici and G. Felici et al., Status of the Cylindrical-GEM project for the KLOE-2 Inner Tracker, Nucl. Instrum. Meth. A 628 (2011) 194 [arXiv:1003.3770 [physics.ins-det]].

[10] G. Amelino-Camelia, F. Archilli, D. Babusci, D. Badoni, G. Bencivenni, J. Bernabeu, R. A. Bertlmann et al., Physics with the KLOE-2 experiment at the upgraded DA $\phi N E$, Eur. Phys. J. C 68 (2010) 619 [arXiv:1003.3868 [hep-ex]]. 